d'ethnoécologie

\section{Revue d'ethnoécologie}

12 | 2017

La Poule. Pratiques d'élevage et histoire culturelle

\title{
Le coq médiéval
}

Jalons pour une histoire symbolique

The medieval cock. Benchmarks for a symbolic history

\section{Michel Pastoureau}

\section{CpenEdition}

\section{Journals}

Édition électronique

URL : http://journals.openedition.org/ethnoecologie/3288

DOI : 10.4000/ethnoecologie.3288

ISSN : 2267-2419

Éditeur

Laboratoire Eco-anthropologie et Ethnobiologie

Référence électronique

Michel Pastoureau, «Le coq médiéval », Revue d'ethnoécologie [En ligne], 12 | 2017, mis en ligne le 18 décembre 2017, consulté le 19 avril 2019. URL : http://journals.openedition.org/ethnoecologie/3288 DOI : 10.4000/ethnoecologie.3288

Ce document a été généré automatiquement le 19 avril 2019

\section{(c)}

Revue d'ethnoécologie est mis à disposition selon les termes de la licence Creative Commons Attribution - Pas d'Utilisation Commerciale - Pas de Modification 4.0 International. 


\title{
Le coq médiéval
}

\author{
Jalons pour une histoire symbolique \\ The medieval cock. Benchmarks for a symbolic history
}

Michel Pastoureau

1 Le discours médiéval sur le coq est en partie l'héritier de celui des auteurs latins de l'Antiquité. Ces derniers ont pour cet oiseau une grande sympathie, voire pour certains une véritable admiration. Attribut de plusieurs divinités (Apollon, Mars, Cérès, Mercure), le coq joue un rôle important dans la divination : on étudie son chant, sa démarche, ses sauts, ses battements d'ailes, son comportement devant la nourriture, et on en tire des conclusions quant à la conduite à tenir ou les décisions à prendre. En milieu militaire, de telles pratiques sont fréquentes pour savoir s'il faut ou non engager le combat. Bien des généraux doivent à un coq leur plus glorieuse victoire. Ce qui pousse Pline, militaire luimême, à écrire dans son Histoire naturelle cette phrase étonnante: «Les coqs sont les maîtres du monde $»^{1}$. C'est en partie à sa crête rouge vif que le coq doit une semblable gloire. Un oiseau qui porte sur sa tête une telle couronne ne peut qu'être aimé des dieux et leur servir de messager. D'autant, nous dit Élien, qu'il met les lions en fuite et qu'il manifeste en toutes occasions une ardeur et une audace indomptables; au point qu'il est parfois nécessaire de le castrer, ajoute malicieusement Isidore de Séville ${ }^{2}$.

2 Héritiers de Pline, d'Élien, d'Isidore et des fabulistes romains, les bestiaires médiévaux, qu'ils soient latins ou vernaculaires, voient eux aussi dans le coq un oiseau courageux et généreux, qui partage sa nourriture avec ses poules, les défend vaillamment et n'hésite pas à affronter des animaux plus forts que lui. Mais puisant aussi à d'autres sources, notamment la Bible et les Pères, bestiaires et volucraires ${ }^{3}$ en font également un oiseau vaniteux, oublieux, sensuel, sinon lubrique. Sous la plume de leurs auteurs, sa symbolique devient ambivalente (Pastoureau 2011 : 156-159).

\section{Le coq des bestiaires}

3 Le courage du coq a maintes fois été célébré par les auteurs de l'Antiquité (Belfiore 2010: 319-324). Les bestiaires, les volucraires et les encyclopédies du Moyen Âge vont plus loin 
et mettent en scène le coq luttant contre le renard, le loup et même le lion. Ce dernier du reste ne craint qu'un seul animal au monde : le coq blanc. Pourquoi blanc? Nos auteurs ne le disent pas (Hambrör 1966: 237-254). Mais ils vantent la hardiesse du coq ${ }^{4}$, sa vigilance constante, l'affection qu'il porte à ses poules et sa largesse, plus généreuse encore que celle du lion. Le coq est partageux : quand il trouve de la nourriture, il appelle ses gélines et leur donne à manger ; à toutes, pas seulement à sa préférée. Cette dernière, en revanche, a le droit, le soir venu, de se coucher près de lui. Parmi ses douze poules, celle qu'il aime le mieux est toujours la plus grosse, la plus grasse, la plus tendre.

4 Lui-même se croit très beau, est fier de ses plumes, de sa crête, de son jabot. Il marche comme un paon et, comme lui, tente de faire la roue. Aux dires des bestiaires, cependant, tous les coqs ne sont pas beaux, tant s'en faut. Certains sont trop petits, d'autres ont les pattes trop courtes, d'autres encore arborent une crête si modeste qu'on peut les prendre pour des poules. Pierre de Crescens nous explique comment doit se présenter un beau coq (Mane 2006: 38) :

« Un beau coq est grand, haut sur ses pattes, large de poitrine. Il doit avoir la voix forte, la crête très rouge, les yeux noirs et hardis, le bec court et pointu, le cou bien doré, les cuisses puissantes et velues, pas trop longues. Ses plumes doivent montrer des couleurs variées, du rouge, du jaune, du noir et même du vert et du bleu, spécialement au niveau de sa queue. Celle-ci doit avoir la forme d'une faucille ou, mieux, d'un croissant de lune ».

Thomas de Cantimpré, lui aussi, insiste sur le lien entre le coq et la lune. Elle a une grande influence sur son comportement : « quand la lune apparaît, le coq se met à sauter comme un possédé $»^{5}$.

6 Toutefois, c'est sur son chant que les bestiaires et les encyclopédies sont les plus bavards. Ce chant n'est en rien celui du cygne, auquel nos auteurs se plaisent parfois à l'opposer. C'est du reste plus un cri qu'un chant, fait de quatre syllabes répétées plutôt que de quatre notes. Comme le coq est un oiseau domestique, ce chant indique les lieux habités, «tout comme le grognement du porc, le braiement de l'âne et le mugissement de la vache» (Hambrör 1966: 243). Il éveille ceux qui dorment et rassure les voyageurs nocturnes. Le coq, en effet, chante à heure fixe, non seulement le jour mais une partie de la nuit. Il veille, et son chant, comme la cloche et mieux que la chandelle, sert à indiquer les heures. Cette notation conduit naturellement tous nos auteurs à rappeler comment, par trois fois, un coq a chanté pour souligner le reniement de Pierre ${ }^{6}$ (Figure 1). 
Figure 1 : Le coq de saint Pierre. Église Notre-Dame-des-Fontaines, La Brigue (Alpes-Maritimes), peint en 1492 par Jean Baleison et Jean Canavesio

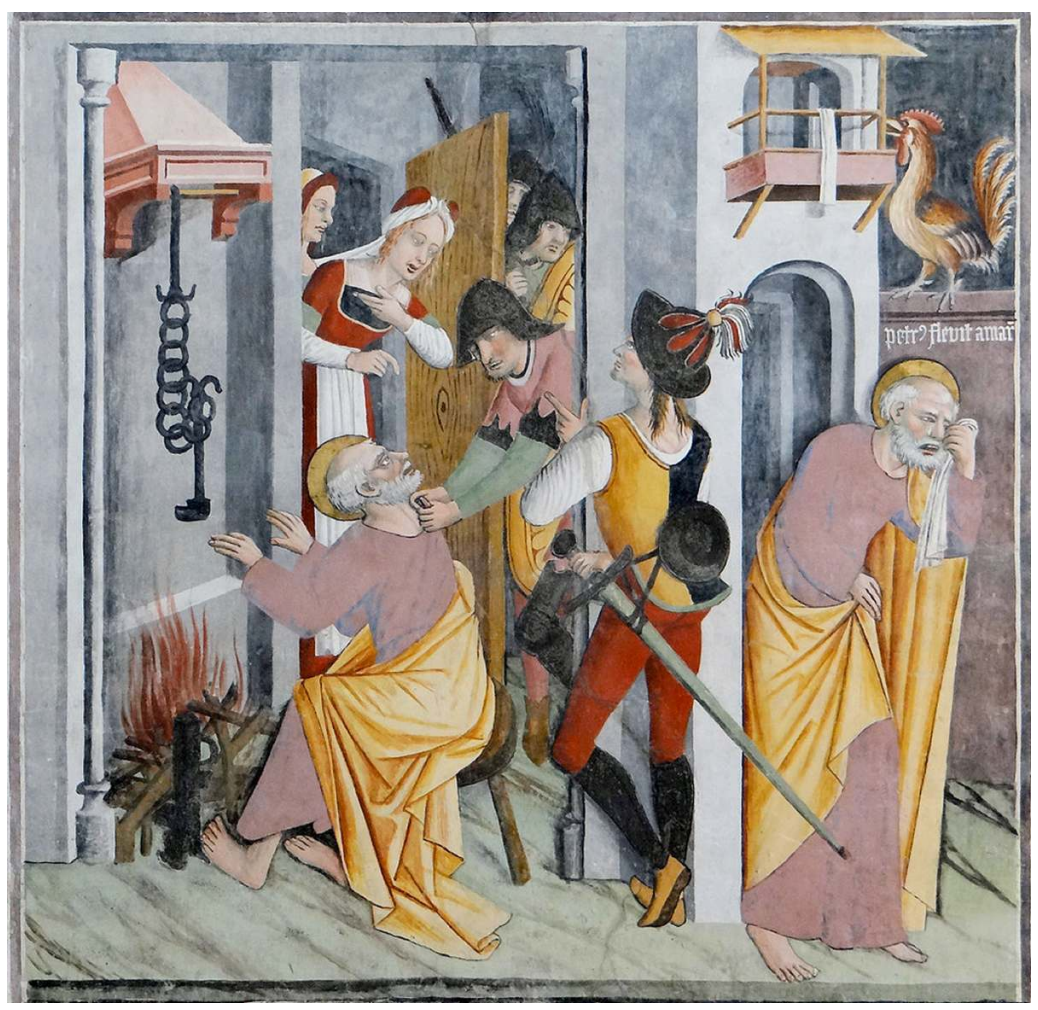

7 Il est l'image du bon pasteur qui invite les chrétiens au repentir et à la pénitence. Il est aussi, dans les images, dès l'époque paléochrétienne, l'attribut de Pierre, rôle qu'il partagera plus tard avec la clef. Les premiers coqs installés aux clochers des églises, dès avant l'an mille, le sont du reste sur des églises dédiées à saint Pierre (Martin 1903-1904). Par la suite, cet usage s'étendra peu à peu à la plupart des églises de la chrétienté romaine, l'oiseau de Pierre devenant l'oiseau vigilant, celui qui surveille les alentours et qui par son chant bénéfique éloigne les forces du mal.

Certains auteurs affirment que le coq chante plus fort la nuit pour mettre en fuite les loups, les renards, les démons et les voleurs. Mais d'autres, plus nombreux, soutiennent que c'est à l'aube qu'il chante avec le plus d'entrain, accompagnant le son de sa voix d'un triple battement d'ailes. Ce faisant, il salue le lever du soleil, invite les hommes à délaisser le sommeil, ranime leur ardeur et leur foi. Il faut se lever, prier Dieu, se mettre au travail. C'est un oiseau vigilant, un envoyé du Seigneur, et le prêtre est à son image qui est comme « le coq de Dieu $»^{7}$.

Plusieurs auteurs comparent le coq tantôt aux moines qui, comme lui, chantent les heures du jour, tantôt aux clercs séculiers, qui veillent sur leurs fidèles comme les coqs veillent sur leurs poules. Un liturgiste de la fin du XIII ${ }^{\mathrm{e}}$ siècle, Guillaume Durand, l'un des auteurs les plus lus jusqu'à la fin du Moyen Âge, ajoute que le coq, «symbole de victoire et de vigilance, possède le pouvoir de chasser les démons ; par son chant : il crie vers Dieu pour hâter l'aurore du Jugement dernier et de la vie éternelle »; c'est pourquoi il apparaît comme l'image «du prêtre cherchant à conduire ses ouailles vers le Salut » (Martin 1903-1904) $)^{8}$. 
Le coq des bestiaires n'a cependant pas que des qualités. C'est aussi, nous apprennent-ils, un oiseau jaloux, qui ne partage pas ses poules avec un congénère mâle. D'où la fréquence des combats de coqs, toujours violents, souvent mortels, parfois humiliants. Quelques auteurs, reprenant leurs prédécesseurs antiques, prétendent que le coq vaincu se laisse couvrir par le vainqueur qui satisfait sur lui son désir avant de s'emparer de ses gélines. Le vaincu en meurt de honte et de chagrin. Tous les coqs ne finissent pas ainsi, mais tous sont dotés d'une forte ardeur sexuelle (Donati 1905) ${ }^{9}$. Chaque fois qu'il s'est adonné à la copulation, le coq pousse un cri triomphant et joyeux; il se repose quelques instants puis recommence avec une autre poule, puis encore avec une autre, et ce, toute la journée. Polygame et fougueux, le coq est parfois dans la symbolique romane l'attribut de la luxure, figurée par un homme chevauchant un coq. Mais il peut aussi symboliser la fécondité ou la fertilité. Innombrables sont les recettes médicinales à base de testicules de coq pour redonner à l'homme sa vigueur perdue : ceux-ci passent pour augmenter le désir, pousser au coït et rendre fertile. Les testicules du taureau, animal bien plus grand, ont la même réputation.

11 Devenu vieux, le coq reste plus distrait, néglige ses poules, perd la mémoire. D'où l'expression, fréquente au Moyen Âge, «avoir une mémoire de vieux coq ", c'est-à-dire être oublieux (Marino Ferro 1996 : 10). Plus grave, le coq âgé se met à pondre des œufs, plus petits et plus ronds que ceux de la poule, et si par malheur ces œufs sont couvés par une bête venimeuse, comme l'aspic ou le dragon, il en sort un animal terrifiant : le basilic, monstrueux coq serpentiforme, à crête blanche (est-ce elle qui fait peur au lion?), dont le corps est entièrement rempli de venin et dont le regard tue.

Les bestiaires sont également bavards sur la poule, qu'ils prennent rarement en mauvaise part. Elle prend de ses poussins un soin extrême, les réchauffe, les nourrit, les surveille, les défend contre le milan et le renard. L'image de la poule abritant ses poussins sous ses ailes est comparée à celle du Christ sur la Croix protégeant les fidèles ou bien, plus tard, à celle de la Vierge couvrant de son immense manteau l'humanité souffrante. Comme la poule, tous deux nous appellent pour mieux nous protéger du péché et du démon.

Si l'on en croit nos auteurs, la poule est en outre un oiseau fécond qui pond plus que tout autre volatile et sait mener ses œufs à terme. Au demeurant, de nombreuses recettes expliquent comment la fermière doit procéder pour ce faire. Pour avoir une belle couvée, le nombre des œufs doit être impair et l'incubation doit commencer entre le neuvième et le onzième jour de la lune croissante. Une telle affirmation n'étonne guère l'historien : en tous domaines, la culture médiévale préfère les nombres impairs. Divisibles par deux, les nombres pairs passent pour imparfaits, corruptibles, néfastes. Plus étrange, en revanche, est l'affirmation selon laquelle des voyageurs auraient vu en Orient des poules plus grandes que les oies et des coqs ayant la taille des autruches. Leurs corps n'ont pas de plumes, précise Marco Polo, mais est couvert de laine comme celui du mouton. Quant à leurs pattes glabres, elles ont le pouvoir de faire fondre l'or (Franklin 1897, t. I : 154-155).

\section{Le coq de la Gaule, puis de la France}

14 Ce sont les Romains qui les premiers ont associé le coq et la Gaule. À l'origine de cette association, un simple jeu de mots : en latin c'est le même terme, gallus, qui désigne à la fois et l'oiseau et l'habitant de la Gaule. Plusieurs auteurs, dont César dans sa Guerre des Gaules, rapprochent ainsi la fougue des guerriers gaulois de la vaillance du coq défendant 
ses poules (Goudineau 2002: 65-66). Plus tard, Suétone compare la voix de l'empereur Néron au chant d'un coq et souligne ironiquement comment le chant d'un tel volatile risque de réveiller les Gaulois ${ }^{10}$. Chez d'autres auteurs latins de l'époque impériale, on rencontre des métaphores semblables qui associent cet oiseau et la Gaule. Mais il ne s'agit pas encore d'un emblème, simplement d'un jeu de mots de lettrés, autorisant diverses images et comparaisons.

La présence de coqs figurés sur divers objets et monuments d'origine gauloise (monnaies, statuettes, pierres sculptées) a fait croire à certains érudits des $\mathrm{XVIII}^{e}$ et $\mathrm{XIX}^{\mathrm{e}}$ siècles que le coq était un emblème de la Gaule indépendante avant même l'arrivée des Romains. Aujourd'hui nous savons qu'il n'en est rien. Non seulement ces coqs mis au jour par l'archéologie ne sont jamais antérieurs au premier siècle avant notre ère, mais ils sont en outre plus Romains (Belfiore 2010 : 319-324) que Gaulois. Le plus souvent, il s'agit de coqs attributs d'une divinité: Apollon, Minerve, Mars et surtout Mercure. Oiseau solaire chantant le cycle des jours et des nuits, le coq joue en effet un rôle important dans la mythologie et dans la religion romaine. Il est associé au soleil et à la victoire, il sert d'oracle en de nombreuses occasions (on observe aussi bien les intonations de son chant que l'ordonnance de ses entrailles...), il est déjà symbole d'éloquence, de vigilance, de fécondité et de lubricité. C'est au culte de Mercure, dieu pour lequel les Gallo-Romains ont toujours eu une dévotion particulière, qu'il est le plus fréquemment associé. La plupart des objets en forme de coq que les archéologues ont trouvés sur le sol de France depuis trois siècles sont des coqs votifs offerts à ce dieu romain du commerce, des arts et de l'éloquence. Messager et intercesseur universel, Mercure a en Gaule pris en charge une bonne partie des propriétés de l'ancien dieu celtique Lug (Duval 1957 : 186-189).

Après la chute de l'Empire romain, le jeu de mots latin entre gallus, le coq, et Gallus, le Gaulois, se fait plus discret. Isidore de Séville (v.570-636), par exemple, père de l'étymologie médiévale, cherche toujours la vérité des êtres et des choses à travers l'histoire des mots qui les désignent. Or il n'établit aucune relation entre le coq et la Gaule, lui préfèrant une étymologie compliquée, mettant en cause la castration fréquente de cet oiseau ${ }^{11}$. Cette étymologie due à un des auteurs les plus lus du haut Moyen Âge est reprise par les encyclopédies et par la littérature zoologique pendant plusieurs siècles et contribue à dévaloriser le coq. Non seulement il se dévirilise mais - ce qui n'avait jamais été le cas dans l'Antiquité - il devient quelque peu ridicule.

En fait, il faut vraiment attendre le $\mathrm{XII}^{\mathrm{e}}$ siècle pour rencontrer de nouveau des textes latins qui s'appuient sur le double sens du terme gallus et associent le coq et la Gaule, devenue entre-temps la France. Mais cette association n'est plus un amusement littéraire, comme c'était le cas à l'époque romaine. Elle est désormais chargée d'une véritable dimension politique et est le fait d'auteurs au service du roi d'Angleterre ou de l'empereur d'Allemagne. Leurs pamphlets, qui comparent le roi de France à un coq, visent à le ridiculiser : comme l'oiseau, il est vaniteux, sot et querelleur; ses entreprises sont maladroites et vouées à l'échec, son royaume est une basse-cour. Ni Louis VII ni Philippe Auguste, les deux rois visés par ces textes, n'ont adopté un coq pour emblème (ce sont au contraire les deux premiers souverains de la France qui ont fait usage de la fleur de lis), et personne dans leur entourage n'a jamais songé à les associer à l'image d'un coq. Cette image est née hors de France et, pendant de longues décennies, c'est uniquement à l'étranger qu'elle a cours pour dénoncer la politique des souverains français. Après l'Angleterre et l'Allemagne, c'est en effet l'Italie gibeline qui, dans la seconde moitié du XIII ${ }^{\mathrm{e}}$ siècle, recourt à l'image du coq pour condamner la politique expansionniste des 
Français partis avec Charles d'Anjou, frère de saint Louis, conquérir un royaume dans le sud de la péninsule. Plusieurs textes mettent ainsi en scène le coq de la France ridiculisé par l'aigle de l'Empire. Cette opposition politique entre l'aigle et le coq (Beaune 1986: 69-80, Pastoureau 1992, vol. 3 : 507-539), née au cœur du Moyen Âge, restera en Europe une constante de la guerre de propagande que se livreront dans les images les emblèmes nationaux jusqu'en plein $\mathrm{xx}^{\mathrm{e}}$ siècle.

Figure 2 : Le coq français et l'aigle allemand

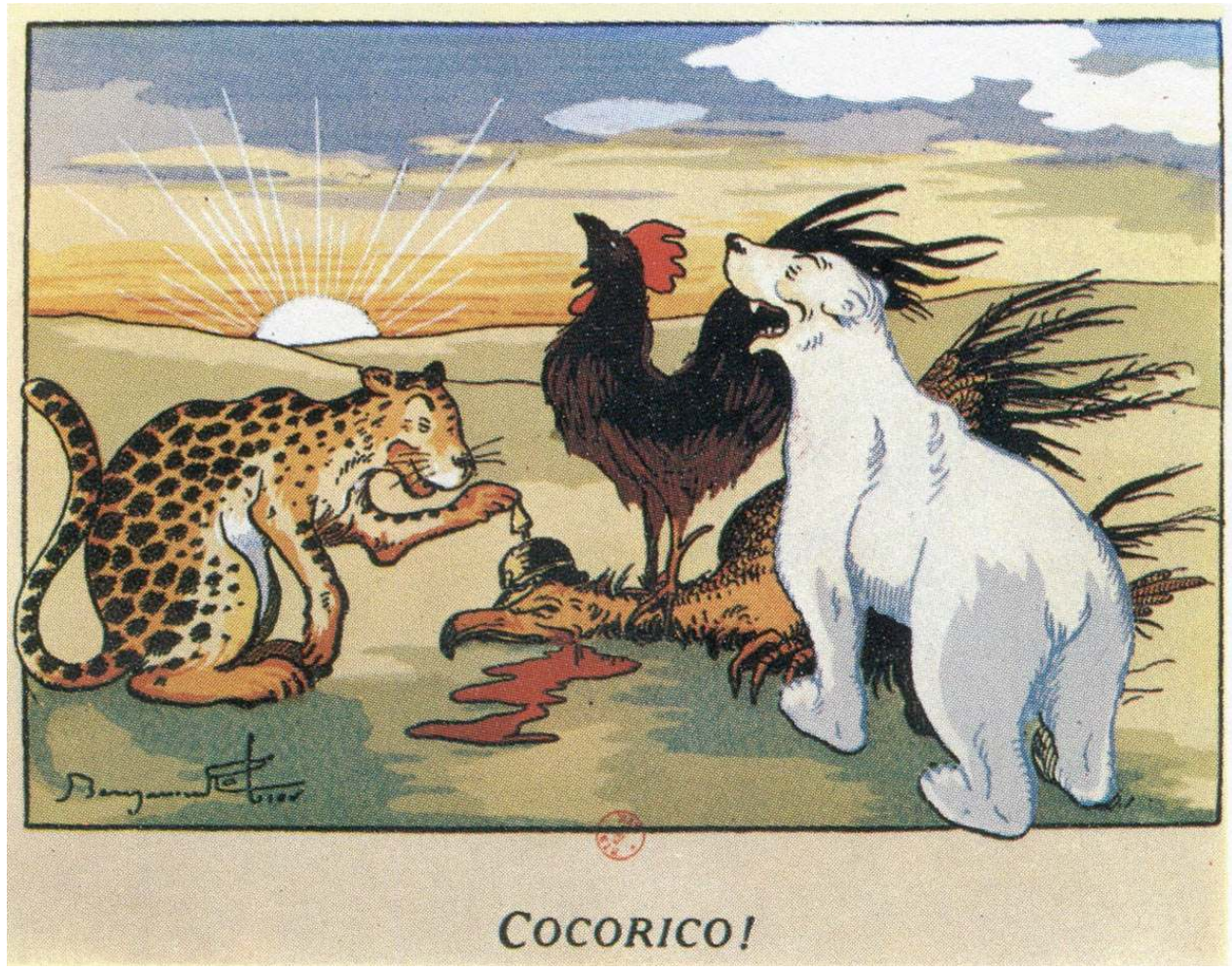

Benjamin Rabier, « Cocorico! » dessin aquarellé au pochoir, 1915 


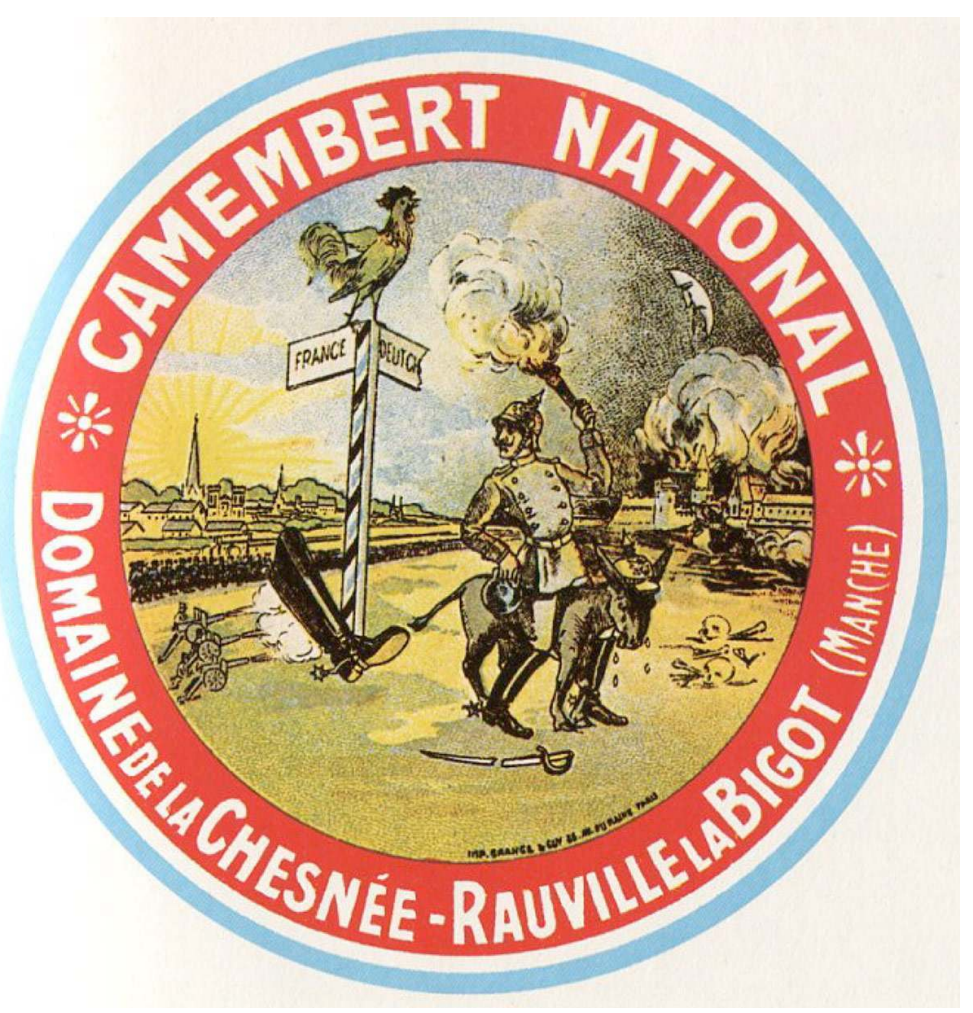

Étiquette de camembert, période 1914-1918

Le recours au jeu de mots latin faisant du coq un emblème ethnique ou politique soulève deux questions. L'une d'ordre linguistique : comment ce jeu de mots peut-il s'exprimer dans la langue vernaculaire ? L'autre d'ordre historique : à partir de quand les habitants du royaume de France ont-ils eu conscience d'avoir pour ancêtres les habitants de la Gaule, les Gaulois? Tentons de répondre d'abord à la première question.

En français moderne, le seul terme d'usage courant pour désigner notre oiseau est le mot coq. D'étymologie obscure, ce mot apparaît au XII ${ }^{\mathrm{e}}$ siècle; mais, jusqu'au XVII ${ }^{\mathrm{e}}$, il reste concurrencé par le mot jal (ou jau), dérivé roman du latin gallus. Ce n'est que sous le règne de Louis XIII (1610-1643) que l'usage de coq semble être devenu plus fréquent que celui de jal, ce dernier terme subsistant cependant, sous des formes variées, dans maints patois et dialectes locaux jusqu'en plein $x^{e}$ siècle (Dupuy 1965). Or, en ancien français comme en français moderne, il n'existe aucune relation apparente, ni phonétique ni sémantique, entre le mot coq et le mot gaulois. La langue vernaculaire, à partir du moment où elle utilise le mot coq pour désigner notre oiseau, ne peut plus exprimer le jeu de mots latin gallus/Gallus. L'historien est donc en droit de se demander par qui et jusqu'à quelle date, hors des milieux lettrés, le lien qui unit le coq et la Gaule reste perceptible. Si l'on n'est pas latiniste ou spécialiste des langues romanes, ce lien est inintelligible. Et ce, aussi bien à la Renaissance que sous Louis XIV, au XVIII ${ }^{\mathrm{e}}$ siècle ou aujourd'hui. Peut-être est-ce là la raison principale (mais il est en d'autres) pour laquelle les Français ont toujours eu du mal à accepter d'être emblématisés par un coq.

D'autant que cet emblème est né d'un simple jeu de mots, presque d'un calembour. Aujourd'hui, sa dimension symbolique peut de ce fait nous sembler faible, voire dérisoire. Il s'agit de ce que l'héraldique appelle une figure " parlante », c'est-à-dire une figure dont 
le choix est simplement dû à une relation entre le nom qui désigne cette figure et le nom de celui qui en fait usage. Dans l'Antiquité et au Moyen Âge, construire un emblème sur une telle relation n'a absolument rien de dévalorisant; bien au contraire, c'est une construction pleine et forte. Mais, à partir du XVII ${ }^{\mathrm{e}}$ siècle, pour des raisons culturelles qui vaudraient la peine d'être étudiées, une telle relation commence à être mal perçue et même jugée vulgaire ou dégradante. Au point que, pendant la Révolution, les partisans du coq gaulois comme emblème national officiel de la France évitent de mettre en avant l'argument linguistique pour justifier leur choix et préfèrent s'en tenir à des raisons historiques, archéologiques ou symboliques. Le jeu de mots latin est passé sous silence. Notre sensibilité contemporaine n'est du reste guère différente de celles des hommes de la fin du XVIII ${ }^{\mathrm{e}}$ siècle : pour nous aussi, faire naître un emblème d'un calembour semble une naissance bâtarde, indigne, suspecte. Et pourtant...

Revenons au XIII ${ }^{\mathrm{e}}$ siècle, au moment où plusieurs auteurs étrangers, écrivant tous en latin, comparent les habitants de la France à des coqs. Leur savoir est purement philologique. Ils savent que les mots Francia et Gallia peuvent être synonymes, mais ils n'ont pas encore vraiment conscience du lien historique qui au fil des âges unit la Gaule antique et la France capétienne. L'opinion la plus courante est alors que l'histoire de la France ne commence qu'au $\mathrm{III}^{\mathrm{e}}$ ou $\mathrm{IV}^{\mathrm{e}}$ siècle de notre ère, lorsque les Francs, descendants supposés des Troyens, commencent à s'installer sur le territoire qui deviendra plus tard le royaume de France. Ce qui se passe avant cette installation est méconnu et n'intéresse guère. En fait, il faut attendre la fin du Moyen Âge pour que commence à être véritablement perçu, sinon compris, le lien qui unit le sol de France et le pays des Gaulois tel qu'en parlent César et les auteurs de l'Antiquité (Beaune 1986 : 67).

Tant que ce lien entre l'histoire de la Gaule et celle de la France n'est pas solidement établi, il est difficile aux Français d'assumer ce coq emblématique dont on les dote à l'étranger. En outre, nous venons de le voir, sa symbolique médiévale est ambivalente, sinon ambiguë. Fables, fabliaux, bestiaires et encyclopédies, après avoir énuméré ses qualités, mettent en valeur ses vices: vaniteux, coléreux, batailleur, fanfaron, sot, lubrique, sensible à la flatterie et à la " vaine gloire »; on le montre souvent victime des autres animaux, tel le Chantecler du Roman de Renart, parfois trompé et violenté par le goupil. Avoir un coq pour emblème est plutôt dégradant.

Les étrangers ont donc beau jeu de comparer le roi de France et ses sujets à des coqs. À partir du XIV e siècle, ils ne le font plus seulement par des textes mais aussi par des images, notamment par les miniatures, plus tard par les médailles et les gravures. Il s'agit presque toujours d'images de propagande dénonçant la politique des Valois hors de France : guerres contre l'Angleterre et la Bourgogne, ambitions italiennes de Charles VIII et de Louis XII, candidature de François I ${ }^{\mathrm{er}}$ au trône impérial.

Toutes ces images ont une vie longue. On les retrouve au XVII ${ }^{e}$ siècle, pendant la guerre de Trente ans ou, plus nombreuses encore, pendant celles de la fin du règne de Louis XIV. Elles mettent en scène un véritable bestiaire politique et emblématique, montrant le coq gaulois aux prises avec l'aigle germanique ou le léopard anglais, voire avec les différents lions de Venise, d'Espagne ou des Provinces-Unies.

Mais, entre-temps, ce coq avait cessé d'être pour les Français un emblème subi, imposé de l'étranger. Il était devenu aussi un emblème assumé, recherché, et finalement glorifié. À la fin du Moyen Âge, en effet, on observe que plusieurs souverains, loin de rejeter le 
volatile auquel les ennemis de la France les associaient, l'ont au contraire accepté et ont retourné à leur avantage la symbolique ambivalente du roi de la basse-cour.

Pour ce faire, les lettrés de leur entourage ont mis au service de la propagande royale non plus le coq stupide, lubrique et vaniteux des fables et du folklore, mais le coq courageux et vigilant de la symbolique chrétienne.

Nombreux sont en effet les textes patristiques et les sermons médiévaux qui, contrairement aux bestiaires, ne prennent le coq qu'en bonne part : chantant le lever du jour, il éloigne les démons de la nuit et réveille les fidèles endormis dans le péché des ténèbres; par là même, il semble les inviter à se repentir et annonce la résurrection future. L'épisode biblique du triple reniement de saint Pierre, tel qu'il est rapporté par les évangiles, sert évidemment d'assise à cette image valorisante du coq.

C'est dans cette symbolique cléricale et christologique du coq que puisent les auteurs au service des rois de France. Christine de Pisan, par exemple, compare Charles V à un coq veillant sur ses sujets. $\mathrm{Au} \mathrm{Xv}^{\mathrm{e}}$ siècle, le surnom de gallus est tour à tour attribué à Charles VII (1422-1461), à Charles VIII (1483-1498) et à Louis XII (1498-1515). Enfin, quelques décennies plus tard, l'entourage de François $I^{\text {er }}$ met en scène un véritable programme politique construit sur la symbolique du coq : lucide, fier, courageux, attribut du soleil, de Mars et de Mercure, emblème générique des anciens Gaulois, le coq est l'image même du roi de France (Lecoq 1987). La mythologie, l'astrologie, l'histoire et l'archéologie sont alors convoquées pour célébrer cet animal qui, au début du Xvi ${ }^{\mathrm{e}}$ siècle, commence à occuper dans l'emblématique royale une place importante, aux côtés de la couronne et de la fleur de lis.

Figure 4 : Le coq blanc de Charles VIII

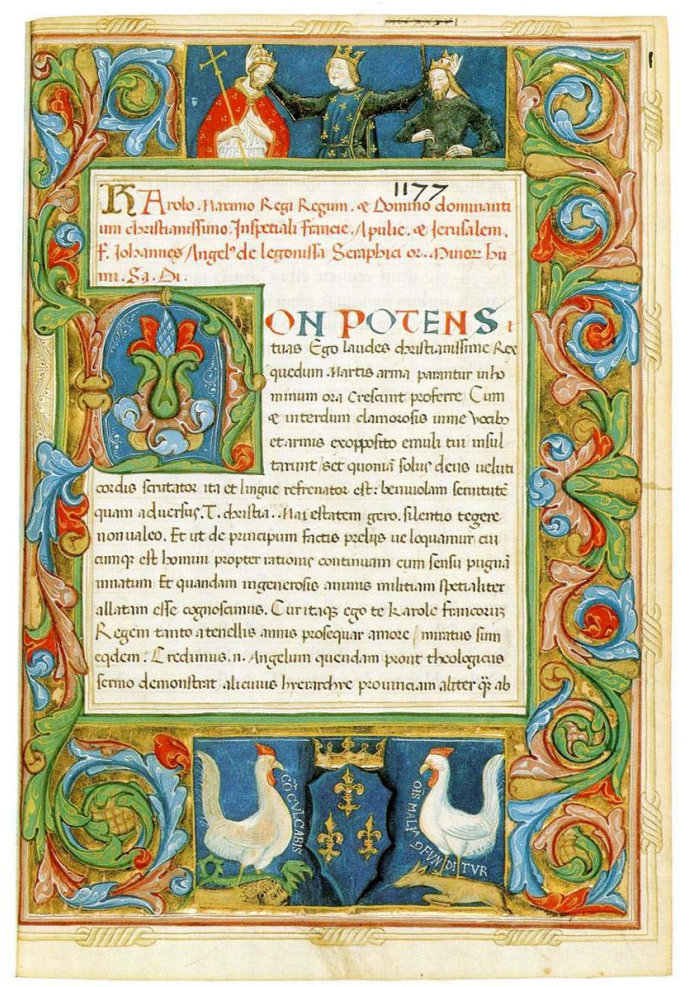

Johannes Angelus Terzo de Legonissa, Opus Davidicum domus Franciae, dédié à Charles VIII par un frère mendiant (sans doute italien, 1495). Paris, BnF, Latin 5971A, f 1 

souvent partie de la propagande monarchique et renvoie tantôt au roi lui-même, tantôt au royaume dans son ensemble, tantôt à la nation française. En 1601, par exemple, à l'occasion de la naissance du futur Louis XIII, son père Henri IV fait couler une médaille sur laquelle on voit le jeune dauphin tenir un sceptre et une fleur de lis tandis que, à ses pieds, un coq, symbole du royaume, pose hardiment la patte sur un globe terrestre. Onze ans plus tard, à l'occasion des fêtes qui célèbrent les fiançailles de ce même Louis XIII avec l'infante Anne d'Autriche, la place Royale à Paris est entièrement décorée de coqs, emblèmes de la France, et de lions, emblèmes de l'Espagne. Ces deux animaux se retrouvent au même endroit en 1660 à l'occasion des fêtes offertes par la ville après le mariage de Louis XIV et de l'infante Marie-Thérèse (Girard 1976 : 126-128).

Cependant, tout au long des guerres du XvII ${ }^{e}$ siècle, ce n'est pas unis mais bien affrontés que ces deux animaux sont mis en scène par les jetons, les médailles et les estampes. Tantôt le coq gaulois effraye le lion espagnol, tantôt au contraire l'oiseau est dévoré par le félin. Et, comme au siècle précédent, ce dernier partage souvent ce repas avec l'aigle impériale, le léopard anglais ou le lion batave. Sur les médailles, l'utilisation de ce bestiaire emblématique à des fins de propagande connaît son apogée pendant les guerres de la Ligue d'Augsbourg (1688-1697). Parallèlement aux opérations militaires se déroule une véritable guerre métallique. Les adversaires de la France retournent contre Louis XIV les allégories dont celui-ci tirait autrefois vanité. En 1692, par exemple, les ProvincesUnies vont jusqu'à faire frapper six médailles pour fêter les défaites navales de la France. Sur l'une d'elles, le soleil se couche tandis que le coq gaulois s'enfuit devant le lion néerlandais ; ce dernier tient dans sa gueule un trident, symbole de sa puissance maritime ${ }^{12}$.

XIV semble avoir eu une attirance particulière pour le thème du coq. Il en fait une figure complémentaire du soleil (son emblème personnel) et des fleurs de lis (emblèmes de la monarchie et de la dynastie). Le coq représente la Nation et, sur plusieurs images, on le voit saluer de son chant un soleil rayonnant. Il apparait alors pleinement comme l'oiseau d'Apollon, c'est-à-dire de Louis XIV lui-même. Mais le roi va plus loin encore : il demande aux artistes qui travaillent à la décoration de Versailles de concevoir un ordre architectural nouveau, l'ordre français, dont les chapiteaux seront destinés à orner plusieurs salles du château. C'est le projet de Lebrun qui est retenu: il fait alterner des coqs et des fleurs de lis. Ce nouvel ordre «français » se voit aujourd'hui encore dans la Galerie des glaces (Maury 1904 : 207-212).

Au XVII ${ }^{e}$ siècle, la promotion artistique et emblématique du coq s'accompagne d'un regain d'intérêt pour les Gaulois. Aux curiosités anecdotiques du siècle précédent succèdent de véritables préoccupations érudites. On écrit beaucoup sur la Gaule et sur les Gaulois, farouches guerriers ancêtres des Français. Le Père Ménestrier, polygraphe infatigable et théoricien influent dans le domaine des emblèmes et des symboles, explique que ces Gaulois, libres et fiers, avaient choisi le coq pour emblème non pas en raison du jeu de mots gallus/Gallus mais bien parce que le coq était par excellence la figure du courage et de la victoire. L'archéologie vient du reste relayer l'érudition : la trouvaille dans le sol de France de chaque objet antique en forme de coq est saluée avec des accents nationalistes (Pastoureau 1992 : 536-53). 


\section{BIBLIOGRAPHIE}

Beaune C. 1986 - Pour une préhistoire du coq gaulois. Médiévales 5 (10) : 69-80.

Belfiore J.-C. 2010 - Dictionnaire des croyances et symboles de l'Antiquité. Paris, Larousse, 1088 p.

Donati A. 1905 - Le dis du koc. Studi medievali : 465-512.

Dupuy A. 1965 - Les noms du coq : jal, jau. Vie et langage $57:$ 87-93.

Duval P.-M. 1957 - Les dieux de la Gaule. Paris, PUF, 136 p.

Franklin A. 1897 - La vie privée d'autrefois. Les animaux. Paris, t. I, 336 p.

Girard A. 1976 - Le coq, personnage de l'histoire. Bourges, A. Girard, 129 p.

Goudineau C. 2002 - Par Toutatis! Que reste-t-il de la Gaule ?. Paris, Le Seuil, 192 p.

Hambrör J. 1966 - Der Hahn als Löwenschreck im Mittelalter. Zeitschrift für Religions- und Geistesgeschichte 18 : 237-254.

Lecoq A.-M. 1987 - François I ${ }^{\text {er }}$ imaginaire. Symbolique et politique à l'aube de la Renaissance française. Paris, Macula, $565 \mathrm{p}$.

Mane P. 2006 - Le travail à la campagne au Moyen Âge. Étude iconographique. Paris, Picard, 471 p.

Marino Ferro X.R. 1996 - Symboles animaux. Un dictionnaire des représentations et croyances en Occident. Paris, Desclée de Brouwer, 484 p.

Martin E. 1903-1904 - Le coq du clocher. Essai d'archéologie et de symbolisme. Mémoires de l'Académie de Stanislas, Nancy : 1-40.

Maury A. 1904 - Les emblèmes et les drapeaux de la France. Le coq gaulois. Paris, Maury, 354 p.

Papin Y.-D. 1993 - Le coq. Histoire, symbole, art, littérature. Paris, Hervas, 134 p.

Pastoureau M. 1992 - Le coq gaulois. In : Nora P. (Ed.), Les Lieux de mémoire. III, Les France.

Paris, Gallimard : 507-539.

Pastoureau M. 2011 - Bestiaires du Moyen Âge. Paris, Seuil, 240 p.

Rey J. 1835 - Histoire du drapeau, des couleurs et des insignes de la monarchie française. Paris, 451 p.

\section{NOTES}

1. Pline, Historia naturalis, livre X, chap. XXIV-XXV.

2. Élien, Variae historiae, Paris, 1602 : 48-49.

3. Un volucraire est un traité sur les oiseaux. Comme dans un bestiaire, les propriétés attribuées à chaque oiseau sont le prétexte à des interprétations morales ou religieuses.

4. La langue du blason qualifie de hardi un coq figuré la patte levée, image qui deviendra à l'époque moderne une enseigne d'auberge «Au coq hardi» et une célèbre marque de camembert dont l'étiquette, en 1942, montre courageusement un coq brochant sur la carte de France et semblant résister à l'envahisseur allemand.

5. Thomas de Cantimpré, Liber de natura rerum, éd. H. Böse, Berlin, 1973, livre 5, chap. LVIII, 205. 
6. Mc 14-30 ; Mt 26-34; Lc 22-34; Jean 13, 38.

7. «Per gallum admonetur prebyter, id est gallus Dei, ut per campanam dormientes ad matiutinas excitet " , écrit Augustin reprenant presque mot à mot Ambroise, Hexamaeron, dans Patrologia latina, vol. 14 , c. 240.

8. Guillaume Durand de Mende, Rationale divinorum officiorum, éd. A. Davril et T. M. Thibodeau, Turnhout, Brepols, 1995, t. I, 19-20.

9. Proverbes et dits le rappellent constamment.

10. Suétone, Vie des douze Césars. Néron, chap. XLV, § 4.

11. Isidore de Séville, Étymologies, livre XII, 7, 50 : «Gallus a castratione vocatus; inter ceteras enim aves huic solo testiculi adimuntur. "

12. Sur cette guerre des médailles : Rey (1835 : 113-137), Papin (1993 : fig. 24-27).

\section{RÉSUMÉS}

Le discours médiéval sur le coq, hérité pour partie des auteurs latins, est diffusé à travers les bestiaires. Sa symbolique ambivalente oscille entre, d'un côté, sa générosité et son courage, de l'autre, sa prétention et sa luxure. Emblème de la Gaule, puis des rois de France à partir du règne de Charles VII (1422-1461), le coq représente la Nation dès celui de Louis XIV, et son image est ensuite régulièrement convoquée à chaque conflit. Lors des deux guerres mondiales, il est l'emblème de la France en lutte contre l'envahisseur.

The medieval speech on the cock, inherited partly from Latin authors, is spread through bestiaries. Its ambivalent symbolism oscillates between, on one side, its generosity and courage, on the other one, its loftiness and its lustful character. An attribute of Gaul, then of the kings of France from the reign of Charles VII (1422-1461), the cock represents the Nation from the reign of Louis XIV, and its image is evoked then regularly in every conflict. During both world wars, it is the emblem of France battling against the invader.

\section{INDEX}

Mots-clés : représentation, symbolique, coq, royauté, nation

Keywords : cock, bestiary, emblem

\section{AUTEUR}

MICHEL PASTOUREAU

EPHE - Directeur d'études 